\title{
Chironomidae collected at the seashore and estuaries in Japan
}

\author{
Koichiro KawaI ${ }^{*, 1)}$, Katsuo Sugimaru ${ }^{2)}$, Hidetoshi SaIto ${ }^{1)}$ and Hiromichi Imabayashi ${ }^{1)}$ \\ * Corresponding author: Koichiro Kawai, Laboratory of Ecology, Graduate School of Biosphere Science, \\ Hiroshima University, 1-4-4, Kagamiyama, Higashihiroshima,Hiroshima 739-8528,Japan \\ (E-mail: kawagogi@hiroshima-u.ac.jp) \\ ${ }^{1)}$ Laboratory of Ecology, Graduate School of Biosphere Science, Hiroshima University, 1-4-4, \\ Kagamiyama, Higashihiroshima, Hiroshima 739-8528, Japan \\ ${ }^{2)}$ Research Dept. Fumakira co.ltd.,1-11-1, Umehara, Hatsukaichi, Hiroshima 739-0494, Japan
}

(Received: 15 March 2011; Accepted: 2 September 2011)

\begin{abstract}
Chironomids collected at seashore and estuaries by bottom sampling of larvae and sweeping and light sampling of adults were compiled during 1994 to 2010 throughout the Japanese Archipelagoes. A total of 87 species of 44 genera in the 4 subfamilies was collected. Eight genera were estimated to comprise marine species additionally to the 17 genera reported to date. Among 3 sampling methods, the highest number of species was collected by light sampling. Ainuyusurika tuberculatum and Cladotanytarsus vanderwulpi were the most widely collected from the Honshu to Yaeyama Islands, whereas Thalassosmittia nemalionis and Thalassomya japonica were collected in the Honshu and Shikoku Islands, and from the Kyushu to Yaeyama Islands, respectively. Tanytarsus boodleae and Yaetanytarsus iriomotensis were collected by all the methods, whereas Clunio tsushimensis was not collected by light sampling, suggesting the lack of phototaxis or weak flying ability of the species.
\end{abstract}

Key words: chironomid, bottom sampling, estuary, seashore, sweeping, light sampling

\section{INTRODUCTION}

Chironomidae (Diptera) comprises about 15,000 species in the world, of which about 2,000 species have been recorded from Japan (Nihon yusurika kenkyu-kai, 2010). It is most widely distributed among the aquatic insects, and is the most abundant in both numbers of species and the amounts in existence (Pinder, 1986). Physiological and ecological studies of some marine species have been reported (Hashimoto, 1975, 1976; Sunose and Fujisawa, 1982). However, the marine chironomid fauna has not been studied after Tokunaga $(1932,1933,1936 a, 1936 b)$ in Japan.

In this study, marine chironomid fauna was conducted to be clarified for coastal regions throughout the Japanese Archipelagoes by three different sampling methods: bottom sampling, sweeping/sucking and light sampling and the differences in distribution range and in collecting methods were discussed among the species.

\section{Materials ANd Methods}

Chironomid samples were collected at the rocky seashore or estuaries of the 65 sites from the Honshu to southwestern islands in Japan in 1994 to 2010. Bottom sampling, sucking/sweeping and light sampling were performed to collect as many species as possible at each site.

Bottom materials, containing larvae, 
comprising green algae grown on the stone, waterweeds, mud, sand and gravels in the intertidal zone near the ebb of the spring tide (from the barnacle zone to waterweed zone) were brought to the laboratory with gentle cooling and constant aeration, and were put into a container $(\Phi 15 \mathrm{~cm} \times \mathrm{H} 9 \mathrm{~cm})$ at $24^{\circ} \mathrm{C}$. A container was filled with $1 / 4$ to $3 / 4$ seawater to $5 \mathrm{~cm}$ level according to the condition of each site, and aerated using a filtration system (Roka Boy Mini, GEX, Osaka). Emerging adults were collected for about 2 months, and males were used for identification. Sucking was performed using a sucking tube $(\Phi 22 \times$ $90 \mathrm{~mm}$ ) for the resting midges on the rocks or buildings. Sweeping was performed with a sweeping net $(\Phi 36 \mathrm{~cm})$ over the rocks and vegetation. Light sampling was usually performed by collecting midges attracted to the light emmited by a variety of vending machines. A portable fluorescent light, equipped with a black lamp (6w, $350 \mathrm{~nm}$ in peak wavelength), was used in case that vending machines were not available.

Wings of males were removed and mounted dry on slide glass, the body was macerated in hot $10 \% \mathrm{KOH}$ solution, washed in water, and mounted in gum-chloral solution.

The specimens were identified under microscope mainly according to Tokunaga (1937), Pinder (1978), Wiederholm (1989), Sasa and Kikuchi (1995) and Saether (2006).

\section{Results AND Discussion}

A total of 87 species of 44 genera in 4 subfamilies was collected (Table 1). Among these, 34, 41 and 63 species were collected by bottom, sweeping/sucking and light samplings, respectively. This shows the highest efficiency of light sampling in species collection among the 3 methods.

Among 87 species, Tanytarsus lamnicaudus Tokunaga, originally described on the specimen collected in the Caroline Islands was firstly collected from Japan in this study. A total of 11 species belonging to 8 genera, Ainuyusurika Sasa et Shirasaka (1 species), Clunio Haliday (3 species), Pontomyia Edwards (1 species), Semiocladius Sublette et Wirth (1 species), Telmatogeton Schiner (2 species), Thalassomya Schiner (1 species), Thalassosmittia Strenzke et Remmert (1 species) and Yaetanytarsus Sasa (1 species) have been described as marine or blackish whereas a total of 17 species in other 9 genera, Chironomus Meigen (1 species), Cladotanytarsus Kieffer (1 species), Cricotopus van der Wulp (1 species), Dicrotendipes Kieffer (3 species), Kiefferulus Goetghebuer (1 species), Limnophyes Eaton (1 species), Polypedilum Kieffer (2 species), Pseudosmittia Goetghebuer (1 species) and Tanytarsus van der Wulp (6 species) have been reported to comprise marine or blackish species (See Table 1; Tokunaga, 1964; Sasa and Kikuchi, 1995; Wiederholm, 1989; Cranston, 2007; Sugimaru et al., 2008). Other 18 species in additional 8 genera, Corynoneura Winnertz (1 species), Cryptochironomus Kieffer (1 species), Eukiefferiella Kieffer (1 species), Microchironomus Kieffer (2 species), Paracladopelma Harnisch (1 species), Paratanytarsus Bause (1 species), Paratrichocladius Santos-Abreu (1 species), Phaenopsectra Kieffer (1 species) to abovementioned 7 genera, Chironomus (1 species), Cladotanytarsus (1 species), Dicrotendipes (2 species), Kiefferulus (1 species), Limnophyes (2 species), Pseudosmittia (1 species) and $\mathrm{Ta}$ nytarsus (1 species) were proven to comprise brackish or marine species by bottom larval 
sampling. Other genera were also shown to be likely to dwell in brackish or marine habitats, although they could comprise those attracted to light sources set near seashores from focal freshwater habitats.

Ainuyusurika tuberculata (Tokunaga), Microchironomus tener (Kieffer), Polypedilum tananense Sasa et Hasegawa, Cladotanytarsus vanderwulpi (Edwards) and Yaetanytarsus iriomotensis Sasa were the most widely collected from the Honshu to Yaeyama Islands, and Cricotopus sylvestris (Fabricius), Paratrichocladius rufiventris (Meigen) and Semiocladius endocladiae (Tokunaga) were also widely collected from the Honshu to Okinawa Islands. In contrast, Thalassosmittia nemalionis (Tokunaga) and Thalassomya japonica Tokunaga et Komyo were collected in the Honshu and Shikoku Islands, and from the Kyushu to Yaeyama Islands, respectively. Pseudorthocladius fujioctavus Sasa, and Hanochironomus tsumerestylus Ree and Kiefferulus glauciventris (Kieffer) were collected in the Satsunan to Okinawa Islands and Okinawa to Yaeyama Islands, respectively. There were some differences in collection area among the species for several genera. For the genus Limnophyes, Limnophyes minimus (Meigen) was widely collected from the Honshu to Yaeyama Islands whereas $L$. natalensis Kieffer was collected only in the Kume and Tokashiki Islands. For the genus Clunio, C. pacificus Edwards and C. setonis Tokunaga were collected in the Kyushu to Yaeyama Islands and the Shikoku, Kyushu and Okinawa Islands, respectively, whereas C. tsushimensis Tokunaga collected in the Honshu, comprising the Sea of Japan and Seto Inland Sea, and Shikoku Islands. For Telmatogeton, T. pacificus Tokunaga was collected only in the Shikoku Island whereas $T$. japonicus Tokunaga collected in the Honshu, comprising the Sea of Japan and Seto Inland Sea, Shikoku and Satsunan Islands. For Dicrotendipes, D. enteromorphae (Tokunaga) was collected from the Honshu, Shikoku, Kyushu to Okinawa Islands whereas D. flexus (Johannsen) was much more widely from the Honshu to Yaeyama Islands. For Tanytarsus, T. boodleae Tokunaga was the most widely collected from the Honshu to Yaeyama Islands, and T. pelagicus Tokunaga was also widely collected from the Honshu, Kyushu to Okinawa Islands whereas T. churamarinus Sugimaru, Kawai et Imabayashi was collected only in the Okinawa and Yaeyama Islands. Of these species, Y. iriomotensis has been recorded only from Iriomote Island until this study.

Pontomyia pacifica Tokunaga, Bryophaenocladius togaflex Sasa et Okazawa and Thalassosmittia nemalionis, and Hanochironomus tsumerestylus were collected only by bottom sampling, sweeping/sucking and light sampling, respectively. For Pontomyia, female adults are well known to be completely devoid of locomotory power and males to only glide on water surface by means of the fluttering wings (Tokunaga, 1932). Besides, most species of Bryophaenocladius larvae are known to be terrestrial or semi-terrestrial. Since Thalassosmittia larvae were reported to be a littoral shore dweller (Wiederholm, 1989), they are possible to live in a little lower zone than our sampling ranges and additionally to have no positive phototaxis. Hanochironomus tsumerestylus was originally described on the samples collected at the Korean coastal village (Ree, 1992) and the larvae were collected from the sand-filter beds in filtration plants located along streams (Yamamoto et al., 2007). 
Semiocladius endocladiae, Telmatogeton japonicus, Thalassomya japonica, Tanytarsus pelagicus etc. were collected only by sweeping/ sucking and light sampling. As the larvae of these species were known to live in the intertidal zone including algal matting (Tokunaga, 1933; Wiederholm, 1989), they might dwell in rather deep zones near the infralittoral one. Clunio tsushimensis was collected only by bottom sampling and sweeping/sucking. This suggests the lack of positive phototaxis and/or a weak flying ability of the species due to their rather atrophied wings (Tokunaga, 1937). Cryptochironomus albofasciatus, Dicrotendipes enteromorphae, Kiefferulus glauciventris etc. were collected only by bottom and light samplings, suggesting the lack of roaring behavior of the adults on or just above the seashore. Paratrichocladius rufiventris, Limnophyes minimus, Ainuyusurika tuberculatus, Cladotanytarsus vanderwulpi, Tanytarsus boodleae etc. were collected by all the methods, suggesting their larval habitats in rather high intertidal zone, a more or less high activity and positive phototaxis of the adults.

\section{REFERENCES}

Cranston, P. S. 2007. The chironomidae larvae associated with the Tsunami-impacted waterbodies of the coastal plains of southwestern Thailand. Raffles Bull. Zool., 55: 231-244.

Hashimoto, H. 1975. Seasonal emergence of Clunio aquilonius Tokunaga (Diptera: Chironomidae). Konty $\bigotimes, 43$ : 52-57.

Hashimoto, H. 1976. Non-biting midges of marine habitats (Diptera: Chironomidae). Marine insects. North Holland Publishing Company, Amsterdam. 377-414.
Nihon yusurika kenkyu-kai. 2010. Illustrated guide to the Chironomidae of Japan. Bun-ichisogoshuppan, Tokyo. 353 pp. (in Japanese)

Pinder, L. C. V. 1978. A key to adult males of British Chironomidae. Part 1. The key; Part 2. Illustrations of the hypopygia. Scient. Publs. Freshwat. Biol. Ass., 37: 169 pp., 189 Figs.

Pinder, L. C. V. 1986. Biology of freshwater Chironomidae. Ann. Rev. Ent., 16:211-230.

Ree, H.-I. 1992. A new genus and new species of Chironomidae from Korea. Jpn. J. Sanit. Zool., 43: 19-22.

Sasa, M. and Kikuchi, M. 1995. Chironomidae [Diptera] of Japan. University of Tokyo Press. 333 pp.

Saether, O. A. 2006. Japanese Pseudosmittia Edwards (Diptera: Chironomidae). Zootaxa, 1198: 21-51.

Sugimaru, K., Kawai, K. and Imabayashi, H. 2008. A new marine chironomid species of the genus Tanytarsus (Diptera: Chironomidae) from Okinawa, Japan. Plankton Benthos Res., 3: 240-242.

Sunose, T. and Fujisawa, T. 1982. Ecological studis of the intertidal chironomid Telmatogeton japonicus Tokunaga in Hokkaido. Res. Popl. Ecol., 24: 70-84.

Tokunaga, M. 1932. Specially evolved chironomids, Pontomyia natans Edwards and P. pacifica Tokunaga, as marine insects. Dobutsugakuzasshi, 44: 1-3.

Tokunaga, M. 1933. Chironomidae from Japan II. Marine Tanytarsus. Philip. J. Sci., 51: 357-367.

Tokunaga, M. 1936a. Chironomidae from Japan VII. New species and a new variety of the genus Chironomus Meigen. Philip. J. Sci., 60: 71-85.

Tokunaga, M. 1936b. Chironomidae from Japan VIII. Marine or seashore Spaniotoma, with description of the immature forms of Spaniotoma melanione, sp. nov. and Tanytarsus boodleae Tokunaga. Philip. J. Sci., 60: 303-319.

Tokunaga, M. 1937. Nihon Dobutsu Bunrui (Fauna Nipponica). Vol. 10, Fasc. 7, No. 1. Family Chironomidae (1). 1-110 (in Japanese)

Tokunaga, M. 1964. Insects of Micronesia Diptera: Chironomidae. Insects of Micronesia, 12: 486-628.

Yamamoto, M., Hirabayashi, K. and Matsuzawa, M. 2007. The Korean species Hanochironomus tumerestylus Ree, 1992 taken on Ishigaki Island, Japan (Diptera: Chironomidae). In: Contribution to the systematics and ecology of aquatic Diptera-a tribute to Ole A. Saether, 2007 (ed. Andersen, T.), pp. 335-346. The Caddis Press.

Wiederholm, T. 1989. Chironomidae from the Holarctic Region-Keys and diagnoses. Entomol. Scand. Suppl., 34, 532 pp. 
Table 1 Records of chironomid collection at the seashores or estuaries throughout the Japanese Archipela goes.

$\bigcirc$, bottom sampling; $\triangle$, sweeping/sucking; $\times$, light sampling.

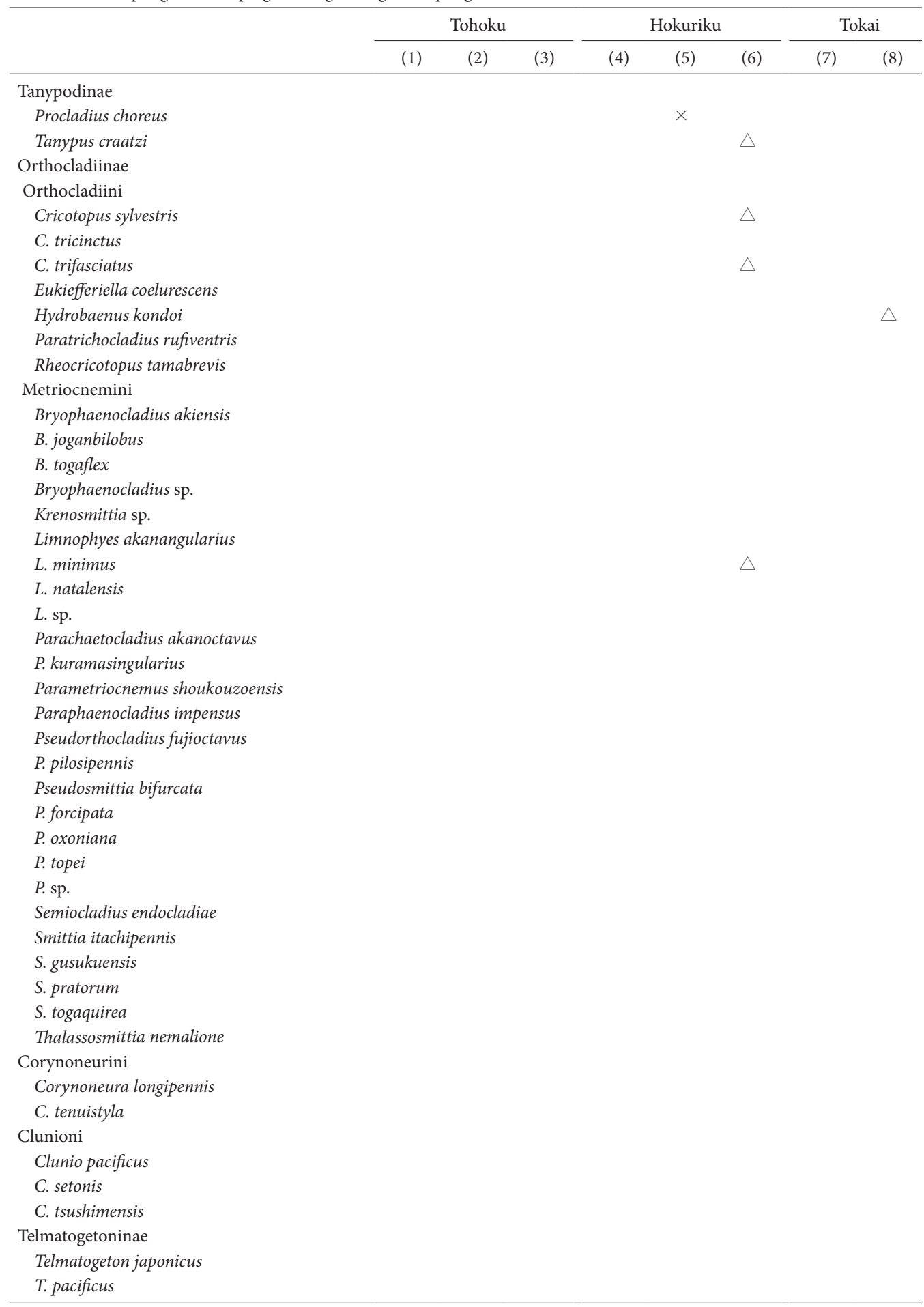
(1) Lake Jusan
(2) Lake Ogawara
(3) Iwaki
(4) Toyama
(5) Shibayamagata
(6) Mikata Lakes
(7) Lake Hamanako

(8) Kuwana 
Table 1 Continued

$\bigcirc$, bottom sampling; $\triangle$, sweeping/sucking; $\times$, light sampling.

\begin{tabular}{|c|c|c|c|c|c|c|c|c|}
\hline & \multicolumn{3}{|c|}{ Tohoku } & \multicolumn{3}{|c|}{ Hokuriku } & \multicolumn{2}{|c|}{ Tokai } \\
\hline & $(1)$ & $(2)$ & (3) & $(4)$ & $(5)$ & $(6)$ & (7) & (8) \\
\hline \multicolumn{9}{|l|}{ Thalassomya japonica } \\
\hline \multicolumn{9}{|l|}{ Chironominae } \\
\hline \multicolumn{9}{|l|}{ Chironomini } \\
\hline \multicolumn{9}{|l|}{ Ainuyusurika tuberculatum } \\
\hline \multicolumn{9}{|l|}{ Chironomus crassiforceps } \\
\hline C. salinarius & & & & $\bigcirc$ & & & $\times$ & \\
\hline \multicolumn{9}{|l|}{ C. sp. } \\
\hline \multicolumn{9}{|c|}{ Cryptochironomus albofasciatus } \\
\hline \multicolumn{9}{|l|}{ Dicrotendipes enteromorphae } \\
\hline D. inouei & & $\times$ & & & & $\bigcirc$ & & \\
\hline \multicolumn{9}{|l|}{ D. lobiger } \\
\hline D. nigrocephalicus & & & $\times$ & & & & & \\
\hline D. pelochloris & & & & & & $\triangle$ & & \\
\hline \multicolumn{9}{|l|}{ D. yaeyamanus } \\
\hline \multicolumn{9}{|c|}{ Hanochironomus tsumerestylus } \\
\hline \multicolumn{9}{|l|}{ Harnischia sp. } \\
\hline \multicolumn{9}{|l|}{ Kiefferulus glauciventris } \\
\hline \multicolumn{9}{|l|}{ K. longilobus } \\
\hline Lipiniella moderata & & & $\times$ & & $\times$ & & & \\
\hline \multicolumn{9}{|l|}{ Microchironomus ishiii } \\
\hline M. tener & & & & & & $\triangle$ & & \\
\hline \multicolumn{9}{|l|}{ M. teruyai } \\
\hline \multicolumn{9}{|l|}{ Nilodrum barbatitarsis } \\
\hline Paracladopelma hibarasecu & & & & & & & & \\
\hline P. tamanipparai & & & & & & & & \\
\hline Phaenopsectra flavipes & & & & & & & & \\
\hline Polypedilum (Pentapedilum & & & & & & & & \\
\hline P. (Pe.) kamosecundum & & & & & & & & \\
\hline P. (Tripodura) tananense & & & & & & & & \\
\hline Tanytarsini & & & & & & & & \\
\hline Cladotanytarsus vanderwul & & & & & & & & \\
\hline C. utonaiquartus & $\times$ & & & & & & & \\
\hline Micropsectra sp. & & & & & & & & \\
\hline Neozavrelia bicoliocula & & & & & & & & \\
\hline N. tamanona & & & & & & & & \\
\hline Paratanytarsus stagnarius & & & & & & & & \\
\hline Pontomyia pacifica & & & & & & & & \\
\hline Rheotanytarsus aestuarius & & & & & & & & \\
\hline R. parvicrinis & & & & & & & & \\
\hline Tanytarsus boodleae & & & & & & & & \\
\hline T. churamarinus & & & & & & & & \\
\hline T. formosanus & & & & & & & & \\
\hline T. lamnicaudus & & & & & & & & \\
\hline T. miyakobrevis & & & & & & & & \\
\hline T. pelagicus & & & & & & & & \\
\hline T. pontophilus & & & & & & & & \\
\hline T. sp. & & & & & & & & \\
\hline Yaetanytarsus iriomotensis & & & & & & & & \\
\hline
\end{tabular}

(1) Lake Jusan (2) Lake Ogawara (3) Iwaki (4) Toyama (5) Shibayamagata (6) Mikata Lakes (7) Lake Hamanako

(8) Kuwana 
Table 1 Continued

$\bigcirc$, bottom sampling; $\triangle$, sweeping/sucking; $\times$, light sampling.

\begin{tabular}{|c|c|c|c|c|c|c|c|c|c|}
\hline & \multicolumn{7}{|c|}{ San-in } & \multicolumn{2}{|c|}{ Kinki } \\
\hline & $(9)$ & (10) & (11) & (12) & (13) & (14) & (15) & (16) & (17) \\
\hline \multicolumn{10}{|l|}{ Tanypodinae } \\
\hline \multicolumn{10}{|l|}{ Procladius choreus } \\
\hline \multicolumn{10}{|l|}{ Tanypus craatzi } \\
\hline \multicolumn{10}{|l|}{ Orthocladiinae } \\
\hline \multicolumn{10}{|l|}{ Orthocladiini } \\
\hline \multicolumn{10}{|l|}{ Cricotopus sylvestris } \\
\hline \multicolumn{10}{|l|}{ C. tricinctus } \\
\hline \multicolumn{10}{|l|}{ C. trifasciatus } \\
\hline \multicolumn{10}{|l|}{ Eukiefferiella coelurescens } \\
\hline \multicolumn{10}{|l|}{ Hydrobaenus kondoi } \\
\hline Paratrichocladius rufiventris & 0 & & & $\bigcirc$ & & & & & \\
\hline \multicolumn{10}{|l|}{ Rheocricotopus tamabrevis } \\
\hline \multicolumn{10}{|l|}{ Metriocnemini } \\
\hline \multicolumn{10}{|l|}{ Bryophaenocladius akiensis } \\
\hline \multicolumn{10}{|l|}{ B. joganbilobus } \\
\hline \multicolumn{10}{|l|}{ B. togaflex } \\
\hline \multicolumn{10}{|l|}{ Bryophaenocladius sp. } \\
\hline \multicolumn{10}{|l|}{ Krenosmittia sp. } \\
\hline Limnophyes akanangularius & & & & & & & & & \\
\hline L. minimus & & & & & & & & & \\
\hline L. natalensis & & & & & & & & & \\
\hline L. sp. & & & & & & & & & \\
\hline Parachaetocladius akanoctavt & & & & & & & & & \\
\hline P. kuramasingularius & & & & & & & & & \\
\hline Parametriocnemus shoukouzc & & & & & & & & & \\
\hline Paraphaenocladius impensus & & & & & & & & & \\
\hline Pseudorthocladius fujioctavus & & & & & & & & & \\
\hline P. pilosipennis & & & & & & & & & \\
\hline Pseudosmittia bifurcata & & & & & & & & & \\
\hline P. forcipata & & & & & & & & & \\
\hline P. oxoniana & & & & & & $\bigcirc$ & & & \\
\hline P. topei & & & & & & & & & \\
\hline P. sp. & & & & & & & & & \\
\hline Semiocladius endocladiae & & & & $\times$ & & & & & $\times \triangle$ \\
\hline Smittia itachipennis & & & & & & & & & \\
\hline S. gusukuensis & & & & & & & & & \\
\hline S. pratorum & & & & & & & & & \\
\hline S. togaquirea & & & & & & & & $\times$ & \\
\hline Thalassosmittia nemalione & & & & & & & & & \\
\hline Corynoneurini & & & & & & & & & \\
\hline Corynoneura longipennis & & & & & & & & & \\
\hline C. tenuistyla & & & & & & & & & \\
\hline Clunioni & & & & & & & & & \\
\hline Clunio pacificus & & & & & & & & & \\
\hline C. setonis & & & & & & & & & \\
\hline C. tsushimensis & & & & & $\triangle$ & & & & \\
\hline Telmatogetoninae & & & & & & & & & \\
\hline Telmatogeton japonicus & & & & & $\triangle$ & & & $x$ & \\
\hline T. pacificus & & & & & & & & & \\
\hline
\end{tabular}

(9) Lake Koyama (10) Lake Togo (11) Lake Nakaumi (12) Lake Shinji (13) Hamada (14) Masuda (15) Susa

(16) Kushimoto (17) Shirahama 
Table 1 Continued

$\bigcirc$, bottom sampling; $\triangle$, sweeping/sucking; $\times$, light sampling.

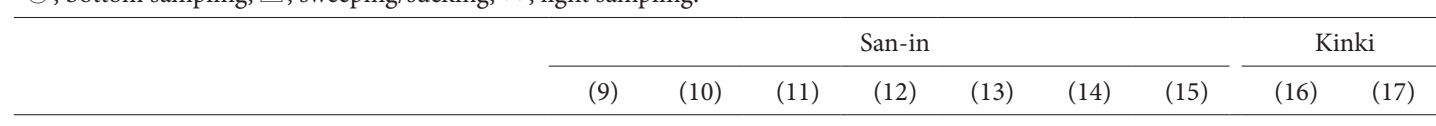

Thalassomya japonica

Chironominae

Chironomini

Ainuyusurika tuberculatum

Chironomus crassiforceps

C. salinarius

C. sp.

Cryptochironomus albofasciatus

Dicrotendipes enteromorphae

D. inouei

D. lobiger

D. nigrocephalicus

D. pelochloris

D. yaeyamanus

Hanochironomus tsumerestylus

Harnischia sp.

Kiefferulus glauciventris

K. longilobus

Lipiniella moderata

Microchironomus ishiii

M. tener

M. teruyai

Nilodrum barbatitarsis

Paracladopelma hibarasecundum

P. tamanipparai

Phaenopsectra flavipes

Polypedilum (Pentapedilum) convexum

P. (Pe.) kamosecundum

$P$. (Tripodura) tananense

Tanytarsini

Cladotanytarsus vanderwulpi

C. utonaiquartus

Micropsectra sp.

Neozavrelia bicoliocula

N. tamanona

Paratanytarsus stagnarius

Pontomyia pacifica

Rheotanytarsus aestuarius

R. parvicrinis

Tanytarsus boodleae

T. churamarinus

T. formosanus

T. lamnicaudus

T. miyakobrevis

T. pelagicus

T. pontophilus

T. sp.

Yaetanytarsus iriomotensis

(9) Lake Koyama (10) Lake Togo (11) Lake Nakaumi (12) Lake Shinji (13) Hamada (14) Masuda (15) Susa

(16) Kushimoto (17) Shirahama 
Table 1 Continued

$\bigcirc$, bottom sampling; $\triangle$, sweeping/sucking; $\times$, light sampling.

Setouchi

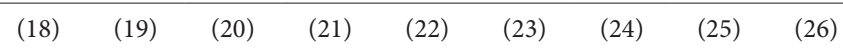

Tanypodinae

Procladius choreus

Tanypus craatzi

Orthocladiinae

Orthocladiini

Cricotopus sylvestris

C. tricinctus

C. trifasciatus

Eukiefferiella coelurescens

Hydrobaenus kondoi

Paratrichocladius rufiventris

Rheocricotopus tamabrevis

Metriocnemini

Bryophaenocladius akiensis

B. joganbilobus

B. togaflex

Bryophaenocladius sp.

Krenosmittia sp.

Limnophyes akanangularius

L. minimus

L. natalensis

L. sp.

Parachaetocladius akanoctavus

P. kuramasingularius

Parametriocnemus shoukouzoensis

Paraphaenocladius impensus

Pseudorthocladius fujioctavus

P. pilosipennis

Pseudosmittia bifurcata

P. forcipata

P. oxoniana

P. topei

P. sp.

Semiocladius endocladiae

Smittia itachipennis

S. gusukuensis

S. pratorum

S. togaquirea

Thalassosmittia nemalione

Corynoneurini

Corynoneura longipennis

C. tenuistyla

Clunioni

Clunio pacificus

C. setonis

C. tsushimensis

Telmatogetoninae

Telmatogeton japonicus

T. pacificus

(18) Lake Kojima (19) Tajiri (20) Mihara (21) Yasuura (22) Kure (23) Ujina (24) Itsukaichi (25) Ohtake

(26) Ikuchi Is. 
Table 1 Continued

$\bigcirc$, bottom sampling; $\triangle$, sweeping/sucking; $\times$, light sampling.

Setouchi

$\begin{array}{llllllllll}(18) & (19) & (20) & \text { (21) } & \text { (22) } & \text { (23) } & \text { (24) } & \text { (25) } & \text { (26) }\end{array}$

Thalassomya japonica

Chironominae

Chironomini

Ainuyusurika tuberculatum

Chironomus crassiforceps

C. salinarius

C. sp.

Cryptochironomus albofasciatus

Dicrotendipes enteromorphae

D. inouei

D. lobiger

D. nigrocephalicus

D. pelochloris

D. yaeyamanus

Hanochironomus tsumerestylus

Harnischia sp.

Kiefferulus glauciventris

K. longilobus

Lipiniella moderata

Microchironomus ishiii

M. tener

M. teruyai

Nilodrum barbatitarsis

Paracladopelma hibarasecundum

P. tamanipparai

Phaenopsectra flavipes

Polypedilum (Pentapedilum) convexum

P. (Pe.) kamosecundum

$P$. (Tripodura) tananense

Tanytarsini

Cladotanytarsus vanderwulpi

C. utonaiquartus

Micropsectra sp.

Neozavrelia bicoliocula

N. tamanona

Paratanytarsus stagnarius

Pontomyia pacifica

Rheotanytarsus aestuarius

R. parvicrinis

Tanytarsus boodleae

T. churamarinus

T. formosanus

T. lamnicaudus

T. miyakobrevis

T. pelagicus

T. pontophilus

T. sp.

Yaetanytarsus iriomotensis

(18) Lake Kojima (19) Tajiri (20) Mihara (21) Yasuura (22) Kure (23) Ujina (24) Itsukaichi (25) Ohtake

(26) Ikuchi Is. 
Table 1 Continued

$\bigcirc$, bottom sampling; $\triangle$, sweeping/sucking; $\times$, light sampling.

\begin{tabular}{|c|c|c|c|c|c|c|c|c|c|}
\hline & \multicolumn{6}{|c|}{ Setouchi } & \multicolumn{3}{|c|}{ Shikoku } \\
\hline & $(27)$ & $(28)$ & $(29)$ & $(30)$ & $(31)$ & $(32)$ & (33) & $(34)$ & $(35)$ \\
\hline \multicolumn{10}{|l|}{ Tanypodinae } \\
\hline \multicolumn{10}{|l|}{ Procladius choreus } \\
\hline \multicolumn{10}{|l|}{ Tanypus craatzi } \\
\hline \multicolumn{10}{|l|}{ Orthocladiinae } \\
\hline \multicolumn{10}{|l|}{ Orthocladiini } \\
\hline \multicolumn{10}{|l|}{ Cricotopus sylvestris } \\
\hline \multicolumn{10}{|l|}{ C. tricinctus } \\
\hline \multicolumn{10}{|l|}{ C. trifasciatus } \\
\hline Eukiefferiella coelurescens & & & & & & & & & 0 \\
\hline \multicolumn{10}{|l|}{ Hydrobaenus kondoi } \\
\hline \multicolumn{10}{|l|}{ Paratrichocladius rufiventris } \\
\hline \multicolumn{10}{|l|}{ Rheocricotopus tamabrevis } \\
\hline \multicolumn{10}{|l|}{ Metriocnemini } \\
\hline \multicolumn{10}{|l|}{ Bryophaenocladius akiensis } \\
\hline B. joganbilobus & & & $x$ & & & & & & \\
\hline \multicolumn{10}{|l|}{ B. togaflex } \\
\hline \multicolumn{10}{|l|}{ Bryophaenocladius sp. } \\
\hline \multicolumn{10}{|l|}{ Krenosmittia sp. } \\
\hline \multicolumn{10}{|l|}{ Limnophyes akanangularius } \\
\hline L. minimus & & & & & & & & & $\times$ \\
\hline L. natalensis & & & & & & & & & \\
\hline L. sp. & & & & & & & & & \\
\hline Parachaetocladius akanocto & & & & & & & & & \\
\hline P. kuramasingularius & & & & & & & & & \\
\hline Parametriocnemus shoukou & & & & & & & & & \\
\hline Paraphaenocladius impenst & & & & & & & & & \\
\hline Pseudorthocladius fujioctav & & & & & & & & & \\
\hline P. pilosipennis & & & & & & & & & $\times$ \\
\hline Pseudosmittia bifurcata & & & & & & & & & \\
\hline P. forcipata & & & & & & & & & \\
\hline P. oxoniana & & & & & & & & & \\
\hline P. topei & & & & & & & & & \\
\hline$P$ sp. & & & & & & & & & \\
\hline Semiocladius endocladiae & & & & $\Delta x$ & & & $x$ & & $\triangle x$ \\
\hline Smittia itachipennis & & & & & & & & & $\times$ \\
\hline S. gusukuensis & & & & & & & & & \\
\hline S. pratorum & & & & & & & $\times$ & & \\
\hline S. togaquirea & & & & & & & & & \\
\hline Thalassosmittia nemalione & & & $\triangle$ & $\triangle$ & & $\triangle$ & & & $\triangle$ \\
\hline Corynoneurini & & & & & & & & & \\
\hline Corynoneura longipennis & & & & & & & & & 0 \\
\hline C. tenuistyla & & & & & & & & & \\
\hline Clunioni & & & & & & & & & \\
\hline Clunio pacificus & & & & & & & & & \\
\hline C. setonis & & & & & & & & & $\times$ \\
\hline C. tsushimensis & & & $\bigcirc \triangle$ & $\triangle$ & & & & & $\bigcirc$ \\
\hline Telmatogetoninae & & & & & & & & & \\
\hline Telmatogeton japonicus & & & $\Delta x$ & & & $\triangle$ & & $\triangle$ & $\triangle$ \\
\hline T. pacificus & & & & & & & & & $\Delta x$ \\
\hline
\end{tabular}

(27) Aba Is. (28) Ohshiba Is. (29) Kamagari Is. (30) Kurahashi Is. (31) Etajima Is. (32) Ninoshima Is. (33) Naruto (34) Muroto (35) Tosashimizu 
Table 1 Continued

$\bigcirc$, bottom sampling; $\triangle$, sweeping/sucking; $\times$, light sampling.

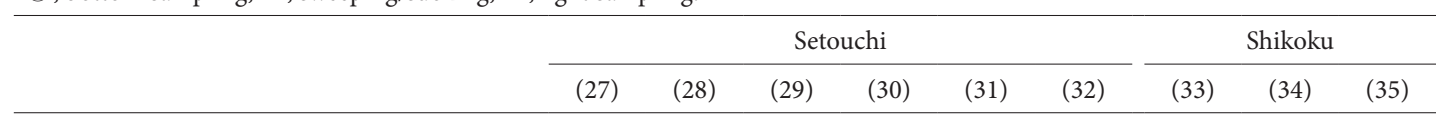

Thalassomya japonica

Chironominae

Chironomini

Ainuyusurika tuberculatum

Chironomus crassiforceps

C. salinarius

C. sp.

Cryptochironomus albofasciatus

Dicrotendipes enteromorphae

D. inouei

D. lobiger

D. nigrocephalicus

D. pelochloris

D. yaeyamanus

Hanochironomus tsumerestylus

Harnischia sp.

Kiefferulus glauciventris

K. longilobus

Lipiniella moderata

Microchironomus ishiii

M. tener

M. teruyai

Nilodrum barbatitarsis

Paracladopelma hibarasecundum

P. tamanipparai

Phaenopsectra flavipes

Polypedilum (Pentapedilum) convexum

P. (Pe.) kamosecundum

$P$. (Tripodura) tananense

Tanytarsini

Cladotanytarsus vanderwulpi

C. utonaiquartus

Micropsectra sp.

Neozavrelia bicoliocula

N. tamanona

Paratanytarsus stagnarius

Pontomyia pacifica

Rheotanytarsus aestuarius

R. parvicrinis

Tanytarsus boodleae

T. churamarinus

T. formosanus

T. lamnicaudus

T. miyakobrevis

T. pelagicus

T. pontophilus

T. sp.

Yaetanytarsus iriomotensis

(27) Aba Is. (28) Ohshiba Is. (29) Kamagari Is. (30) Kurahashi Is. (31) Etajima Is. (32) Ninoshima Is. (33) Naruto (34) Muroto (35) Tosashimizu 
Table 1 Continued

$\bigcirc$, bottom sampling; $\triangle$, sweeping/sucking; $\times$, light sampling.

Satsunan Islands

$\begin{array}{lllllll}(36) \quad(37) \quad(38) & (39) & (40) & (41) & (42) & \text { (43) }\end{array}$

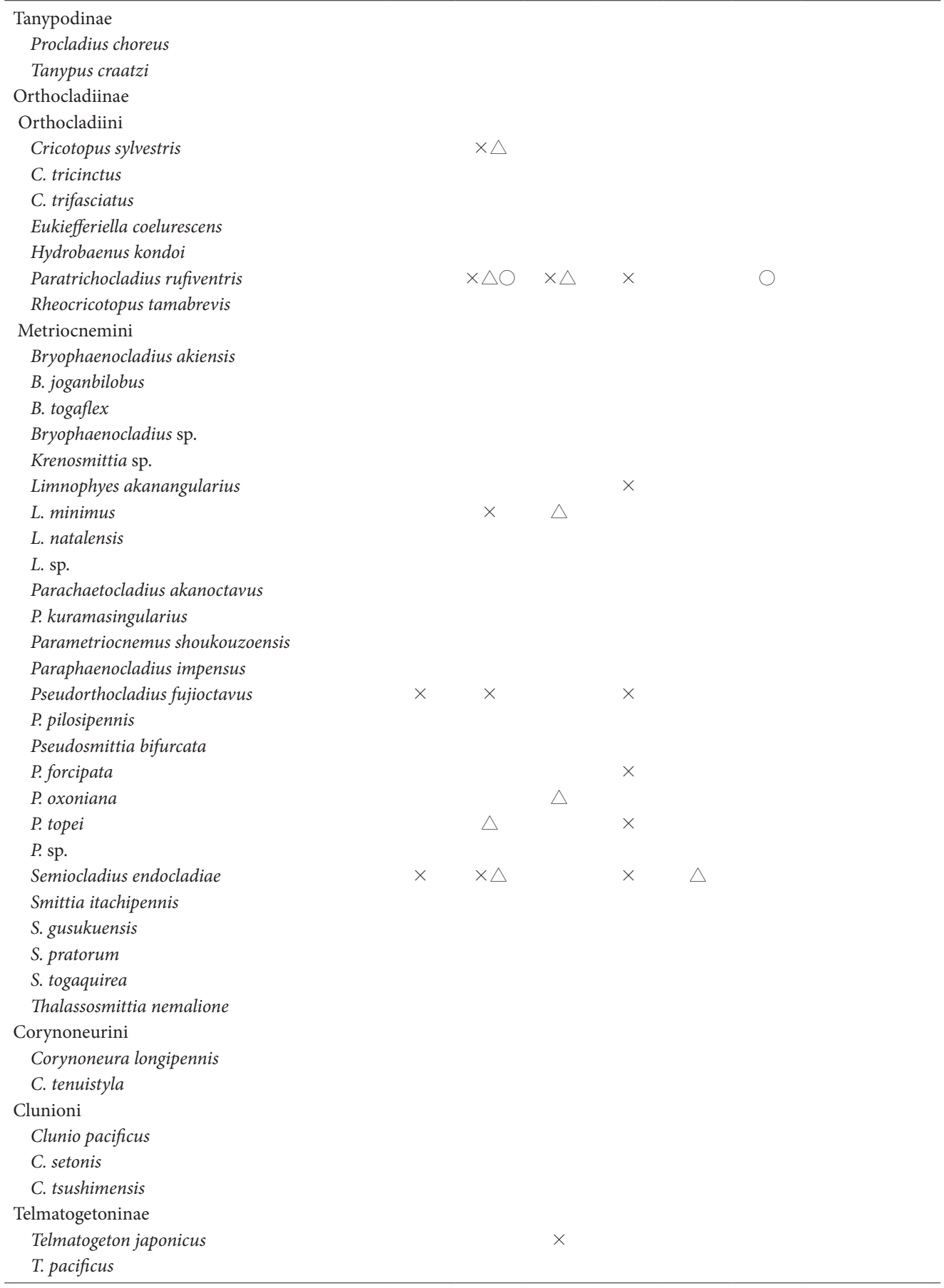

(36) Kuro Is. (Satsuma) (37) Tanegashima Is. (38) Yakushima Is. (39) Amami Is. (40) Kakeroma Is. (41) Kikai Is. (42) Tokunoshima Is. (43) Yoron Is. 
Table 1 Continued

$\bigcirc$, bottom sampling; $\triangle$, sweeping/sucking; $\times$, light sampling.

\begin{tabular}{|c|c|c|c|c|c|c|c|c|}
\hline & \multicolumn{8}{|c|}{ Satsunan Islands } \\
\hline & $(36)$ & $(37)$ & $(38)$ & $(39)$ & $(40)$ & $(41)$ & $(42)$ & $(43)$ \\
\hline Thalassomya japonica & & & & $\triangle$ & & & & \\
\hline \multicolumn{9}{|l|}{ Chironominae } \\
\hline \multicolumn{9}{|l|}{ Chironomini } \\
\hline Ainuyusurika tuberculatum & & $\bigcirc$ & $\times 0$ & & & $\times$ & 0 & \\
\hline \multicolumn{9}{|l|}{ Chironomus crassiforceps } \\
\hline \multicolumn{9}{|l|}{ C. salinarius } \\
\hline \multicolumn{9}{|l|}{ C. sp. } \\
\hline \multicolumn{9}{|l|}{ Cryptochironomus albofasciatus } \\
\hline Dicrotendipes enteromorphae & & & 0 & & & $x$ & & \\
\hline D. inouei & & $\times 0$ & & & & & & \\
\hline \multicolumn{9}{|l|}{ D. lobiger } \\
\hline D. nigrocephalicus & & $\times$ & $\times 0$ & $x$ & & & & \\
\hline \multicolumn{9}{|l|}{ D. pelochloris } \\
\hline \multicolumn{9}{|l|}{ D. yaeyamanus } \\
\hline \multicolumn{9}{|l|}{ Hanochironomus tsumerestylus } \\
\hline \multicolumn{9}{|l|}{ Harnischia sp. } \\
\hline \multicolumn{9}{|l|}{ Kiefferulus glauciventris } \\
\hline \multicolumn{9}{|l|}{ K. longilobus } \\
\hline \multicolumn{9}{|l|}{ Lipiniella moderata } \\
\hline \multicolumn{9}{|l|}{ Microchironomus ishiii } \\
\hline \multicolumn{9}{|l|}{ M. tener } \\
\hline \multicolumn{9}{|l|}{ M. teruyai } \\
\hline \multicolumn{9}{|l|}{ Nilodrum barbatitarsis } \\
\hline Paracladopelma hibarasecundum & & $\times$ & & & & & & \\
\hline \multicolumn{9}{|l|}{ P. tamanipparai } \\
\hline Phaenopsectra flavipes & & & & & & & & \\
\hline Polypedilum (Pentapedilum) conv & & & & & & & & \\
\hline P. (Pe.) kamosecundum & & & & & & & & \\
\hline P. (Tripodura) tananense & & & & & & & & \\
\hline Tanytarsini & & & & & & & & \\
\hline Cladotanytarsus vanderwulpi & & $\times 0$ & & $\bigcirc$ & O & & & \\
\hline C. utonaiquartus & & & & $x$ & & & & \\
\hline Micropsectra sp. & & & & & & & & \\
\hline Neozavrelia bicoliocula & & & & & & & & \\
\hline N. tamanona & $\times$ & & & & & & & \\
\hline Paratanytarsus stagnarius & & & & & & & & \\
\hline Pontomyia pacifica & & & & & & & & \\
\hline Rheotanytarsus aestuarius & & & & $x$ & & & & \\
\hline R. parvicrinis & & & & & & & & \\
\hline Tanytarsus boodleae & & $\times$ & & & & $\times$ & & \\
\hline T. churamarinus & & & & & & & & \\
\hline T. formosanus & & $\triangle$ & & & & & & $\bigcirc$ \\
\hline T. lamnicaudus & & & & & & & & \\
\hline T. miyakobrevis & & & $\bigcirc$ & & & & & \\
\hline T. pelagicus & & & & & & & & \\
\hline T. pontophilus & & & & & & & & \\
\hline T. sp. & & & & & & & & \\
\hline Yaetanytarsus iriomotensis & & $x$ & $x$ & $x$ & & $x$ & & \\
\hline
\end{tabular}

(36) Kuro Is. (Satsuma) (37) Tanegashima Is. (38) Yakushima Is. (39) Amami Is. (40) Kakeroma Is. (41) Kikai Is. (42) Tokunoshima Is. (43) Yoron Is. 
Table 1 Continued

$\bigcirc$, bottom sampling; $\triangle$, sweeping/sucking; $\times$, light sampling.

\begin{tabular}{|c|c|c|c|c|c|c|c|c|}
\hline & \multicolumn{8}{|c|}{ Okinawa Islands } \\
\hline & $(44)$ & $(45)$ & $(46)$ & $(47)$ & $(48)$ & $(49)$ & $(50)$ & $(51)$ \\
\hline \multicolumn{9}{|l|}{ Tanypodinae } \\
\hline \multicolumn{9}{|l|}{ Procladius choreus } \\
\hline \multicolumn{9}{|l|}{ Tanypus craatzi } \\
\hline \multicolumn{9}{|l|}{ Orthocladiinae } \\
\hline \multicolumn{9}{|l|}{ Orthocladiini } \\
\hline Cricotopus sylvestris & $\times$ & & $\bigcirc$ & & & & & \\
\hline \multicolumn{9}{|l|}{ C. tricinctus } \\
\hline \multicolumn{9}{|l|}{ C. trifasciatus } \\
\hline \multicolumn{9}{|l|}{ Eukiefferiella coelurescens } \\
\hline \multicolumn{9}{|l|}{ Hydrobaenus kondoi } \\
\hline Paratrichocladius rufiventris & & & $\bigcirc$ & & & & & \\
\hline Rheocricotopus tamabrevis & & & $\triangle$ & & & $x$ & & \\
\hline \multicolumn{9}{|l|}{ Metriocnemini } \\
\hline Bryophaenocladius akiensis & & & & & & $x$ & & \\
\hline \multicolumn{9}{|l|}{ B. joganbilobus } \\
\hline \multicolumn{9}{|l|}{ B. togaflex } \\
\hline Bryophaenocladius sp. & & & & & $\triangle$ & & & \\
\hline \multicolumn{9}{|l|}{ Krenosmittia sp. } \\
\hline \multicolumn{9}{|l|}{ Limnophyes akanangularius } \\
\hline L. minimus & & & $\triangle 0$ & & & & & \\
\hline L. natalensis & & & & & & 0 & & 0 \\
\hline \multicolumn{9}{|l|}{ L. sp. } \\
\hline \multicolumn{9}{|l|}{ Parachaetocladius akanoctavus } \\
\hline \multicolumn{9}{|l|}{ P. kuramasingularius } \\
\hline \multicolumn{9}{|c|}{ Parametriocnemus shoukouzoensis } \\
\hline Paraphaenocladius impensus & & $\triangle$ & & & & & & \\
\hline Pseudorthocladius fujioctavus & & & $\times$ & & & & & \\
\hline P. pilosipennis & & & & & & & & \\
\hline Pseudosmittia bifurcata & & & & $\triangle$ & & & & \\
\hline P. forcipata & & & & & & & & \\
\hline P. oxoniana & & & & & & & & \\
\hline P. topei & & & & & & $x$ & & \\
\hline P. sp. & & & & & & & & \\
\hline Semiocladius endocladiae & & $\times$ & $\times$ & & & & $\times \triangle$ & \\
\hline Smittia itachipennis & & & & & & & & \\
\hline S. gusukuensis & & & $x$ & & & & & \\
\hline S. pratorum & & & & & & & & \\
\hline S. togaquirea & & & & & & & & \\
\hline Thalassosmittia nemalione & & & & & & & & \\
\hline Corynoneurini & & & & & & & & \\
\hline Corynoneura longipennis & & & & & & & & \\
\hline C. tenuistyla & & & & & & & & \\
\hline Clunioni & & & & & & & & \\
\hline Clunio pacificus & & & & & & & & \\
\hline C. setonis & & & $\triangle$ & & & & & \\
\hline C. tsushimensis & & & & & & & & \\
\hline Telmatogetoninae & & & & & & & & \\
\hline Telmatogeton japonicus & & & & & & & & \\
\hline T. pacificus & & & & & & & & \\
\hline
\end{tabular}

(44) Iheya Is. (45) Izena Is. (46) Okinawa Is. (47) Ie Is. (48) Aguni Is. (49) Kume Is. (50) Zamami Is.

(51) Tokashiki Is. 
Table 1 Continued

$\bigcirc$, bottom sampling; $\triangle$, sweeping/sucking; $\times$, light sampling.

\begin{tabular}{|c|c|c|c|c|c|c|c|c|}
\hline & \multicolumn{8}{|c|}{ Okinawa Islands } \\
\hline & $(44)$ & $(45)$ & $(46)$ & $(47)$ & $(48)$ & $(49)$ & $(50)$ & $(51)$ \\
\hline Thalassomya japonica & & & & & & & $\triangle$ & $\times$ \\
\hline \multicolumn{9}{|l|}{ Chironominae } \\
\hline \multicolumn{9}{|l|}{ Chironomini } \\
\hline Ainuyusurika tuberculatum & & & $\times$ & & & $\times$ & & \\
\hline Chironomus crassiforceps & & $\triangle$ & & & & & & \\
\hline \multicolumn{9}{|l|}{ C. salinarius } \\
\hline \multicolumn{9}{|l|}{ C. sp. } \\
\hline Cryptochironomus albofasciatus & $x$ & $\bigcirc$ & $\times$ & & & & & \\
\hline Dicrotendipes enteromorphae & & $\bigcirc$ & & & & $x$ & & \\
\hline D. inouei & & & & & & $x$ & $x$ & \\
\hline \multicolumn{9}{|l|}{ D. lobiger } \\
\hline D. nigrocephalicus & & & $\times 0$ & & & $\times$ & & \\
\hline \multicolumn{9}{|l|}{ D. pelochloris } \\
\hline D. yaeyamanus & & & 0 & & & & & \\
\hline Hanochironomus tsumerestylus & & & $\times$ & & & & & \\
\hline \multicolumn{9}{|l|}{ Harnischia sp. } \\
\hline Kiefferulus glauciventris & & & 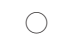 & & & & & \\
\hline \multicolumn{9}{|l|}{ K. longilobus } \\
\hline \multicolumn{9}{|l|}{ Lipiniella moderata } \\
\hline \multicolumn{9}{|l|}{ Microchironomus ishiii } \\
\hline M. tener & & & & & $\triangle$ & & $\times$ & \\
\hline \multicolumn{9}{|l|}{ M. teruyai } \\
\hline \multicolumn{9}{|l|}{ Nilodrum barbatitarsis } \\
\hline \multicolumn{9}{|l|}{ Paracladopelma hibarasecundum } \\
\hline \multicolumn{9}{|l|}{ P. tamanipparai } \\
\hline \multicolumn{9}{|l|}{ Phaenopsectra flavipes } \\
\hline Polypedilum (Pentapedilum) convexum & & & $O \times$ & & & & & \\
\hline \multicolumn{9}{|l|}{ P. (Pe.) kamosecundum } \\
\hline \multicolumn{9}{|l|}{ P. (Tripodura) tananense } \\
\hline \multicolumn{9}{|l|}{ Tanytarsini } \\
\hline Cladotanytarsus vanderwulpi & $\times \bigcirc$ & & & & & & & \\
\hline \multicolumn{9}{|l|}{ C. utonaiquartus } \\
\hline Micropsectra sp. & & & & & & & & \\
\hline Neozavrelia bicoliocula & & & & & & & & \\
\hline N. tamanona & & & & & & & & \\
\hline Paratanytarsus stagnarius & & & & & & & & \\
\hline Pontomyia pacifica & & & & & & & & \\
\hline Rheotanytarsus aestuarius & & & & & & & & \\
\hline R. parvicrinis & & & & & & $\times$ & & \\
\hline Tanytarsus boodleae & & & $\times$ & & $x$ & $x$ & & \\
\hline T. churamarinus & & & & & & $x$ & $\times$ & \\
\hline T. formosanus & $\times$ & & $\times 0$ & & & & & \\
\hline T. lamnicaudus & & & & & & & & \\
\hline T. miyakobrevis & & & & & & & & \\
\hline T. pelagicus & & & & $\triangle$ & & & & \\
\hline T. pontophilus & & & & & & & & \\
\hline T. sp. & & & & & & & & \\
\hline Yaetanytarsus iriomotensis & $x$ & $\times$ & $\times \bigcirc$ & & $x$ & $\times$ & $\times$ & \\
\hline
\end{tabular}

(44) Iheya Is. (45) Izena Is. (46) Okinawa Is. (47) Ie Is. (48) Aguni Is. (49) Kume Is. (50) Zamami Is. (51) Tokashiki Is. 
Table 1 Continued

$\bigcirc$, bottom sampling; $\triangle$, sweeping/sucking; $\times$, light sampling.

\begin{tabular}{|c|c|c|c|c|c|c|c|c|c|}
\hline & \multicolumn{8}{|c|}{ Yaeyama Islands } & \multirow{2}{*}{$\frac{\text { Izu Islands }}{(60)}$} \\
\hline & $(52)$ & $(53)$ & $(54)$ & $(55)$ & $(56)$ & $(57)$ & $(58)$ & $(59)$ & \\
\hline \multicolumn{10}{|l|}{ Tanypodinae } \\
\hline \multicolumn{10}{|l|}{ Procladius choreus } \\
\hline \multicolumn{10}{|l|}{ Tanypus craatzi } \\
\hline \multicolumn{10}{|l|}{ Orthocladiinae } \\
\hline \multicolumn{10}{|l|}{ Orthocladiini } \\
\hline \multicolumn{10}{|l|}{ Cricotopus sylvestris } \\
\hline \multicolumn{10}{|l|}{ C. tricinctus } \\
\hline \multicolumn{10}{|l|}{ C. trifasciatus } \\
\hline \multicolumn{10}{|l|}{ Eukiefferiella coelurescens } \\
\hline \multicolumn{10}{|l|}{ Hydrobaenus kondoi } \\
\hline \multicolumn{10}{|l|}{ Paratrichocladius rufiventris } \\
\hline \multicolumn{10}{|l|}{ Rheocricotopus tamabrevis } \\
\hline \multicolumn{10}{|l|}{ Metriocnemini } \\
\hline \multicolumn{10}{|l|}{ Bryophaenocladius akiensis } \\
\hline \multicolumn{10}{|l|}{ B. joganbilobus } \\
\hline B. togaflex & & & $\triangle$ & & & & $\triangle$ & & \\
\hline \multicolumn{10}{|l|}{ Bryophaenocladius sp. } \\
\hline Krenosmittia sp. & & & & & & $\times$ & & & \\
\hline \multicolumn{10}{|l|}{ Limnophyes akanangularius } \\
\hline L. minimus & & & & $x$ & & & & & \\
\hline L. natalensis & & & & & & & & & \\
\hline L. sp. & & & & & & $\times$ & & & \\
\hline Parachaetocladius akanoctavus & & & & & & & & & \\
\hline P. kuramasingularius & & & & & & & & & \\
\hline Parametriocnemus shoukouzoensis & & & & $\times$ & & & & & \\
\hline Paraphaenocladius impensus & & & & & & & & & \\
\hline Pseudorthocladius fujioctavus & & & & & & & & & \\
\hline P. pilosipennis & & & & & & & & & \\
\hline Pseudosmittia bifurcata & & & & & & & & & \\
\hline P. forcipata & & & & & & & & & \\
\hline P. oxoniana & & & & & & & & & \\
\hline P. topei & & & & $\times$ & & $\times$ & & & \\
\hline P. sp. & & & & & & $\times$ & & & \\
\hline Semiocladius endocladiae & & & & & & & & & \\
\hline Smittia itachipennis & & & & $\times$ & & $\times$ & & & $\triangle$ \\
\hline S. gusukuensis & & & & & & & & & \\
\hline S. pratorum & & & & $\times$ & & & & & \\
\hline S. togaquirea & & & & & & & & & \\
\hline Thalassosmittia nemalione & & & & & & & & & \\
\hline Corynoneurini & & & & & & & & & \\
\hline Corynoneura longipennis & & & & & & & & & \\
\hline C. tenuistyla & & & & & & & & & \\
\hline Clunioni & & & & & & & & & \\
\hline Clunio pacificus & & & & & & $\triangle$ & & & \\
\hline C. setonis & & & & & & & & & \\
\hline C. tsushimensis & & & & & & & & & \\
\hline Telmatogetoninae & & & & & & & & & \\
\hline Telmatogeton japonicus & & & & & & & & & \\
\hline T. pacificus & & & & & & & & & \\
\hline
\end{tabular}

(52) Miyako Is. (53) Irabu Is. (54) Shimoji Is. (55) Ishigaki Is. (56) Yonakuni Is. （57) Iriomote Is. (58) Kuro Is.(Yaeyama)

(59) Hateruma Is. (60) Hachijo Is. 
Table 1 Continued

$\bigcirc$, bottom sampling; $\triangle$, sweeping/sucking; $\times$, light sampling.

\begin{tabular}{cccccccccccc}
\hline \multicolumn{1}{c}{ Yaeyama Islands } & \multicolumn{3}{c}{ Izu Islands } \\
\cline { 2 - 9 } & $(52)$ & $(53)$ & $(54)$ & $(55)$ & $(56)$ & $(57)$ & $(58)$ & $(59)$ & $(60)$ \\
\hline
\end{tabular}

Thalassomya japonica

Chironominae

Chironomini

Ainuyusurika tuberculatum

Chironomus crassiforceps

C. salinarius

C. sp.

Cryptochironomus albofasciatus

Dicrotendipes enteromorphae

D. inouei

D. lobiger

D. nigrocephalicus

D. pelochloris

D. yaeyamanus

Hanochironomus tsumerestylus

Harnischia sp.

Kiefferulus glauciventris

K. longilobus

Lipiniella moderata

Microchironomus ishiii

M. tener

M. teruyai

Nilodrum barbatitarsis

Paracladopelma hibarasecundum

P. tamanipparai

Phaenopsectra flavipes

Polypedilum (Pentapedilum) convexum

P. (Pe.) kamosecundum

$P$. (Tripodura) tananense

Tanytarsini

Cladotanytarsus vanderwulpi

C. utonaiquartus

Micropsectra sp.

Neozavrelia bicoliocula

N. tamanona

Paratanytarsus stagnarius

Pontomyia pacifica

Rheotanytarsus aestuarius

R. parvicrinis

Tanytarsus boodleae

T. churamarinus

T. formosanus

T. lamnicaudus

T. miyakobrevis

T. pelagicus

T. pontophilus

T. sp.

Yaetanytarsus iriomotensis

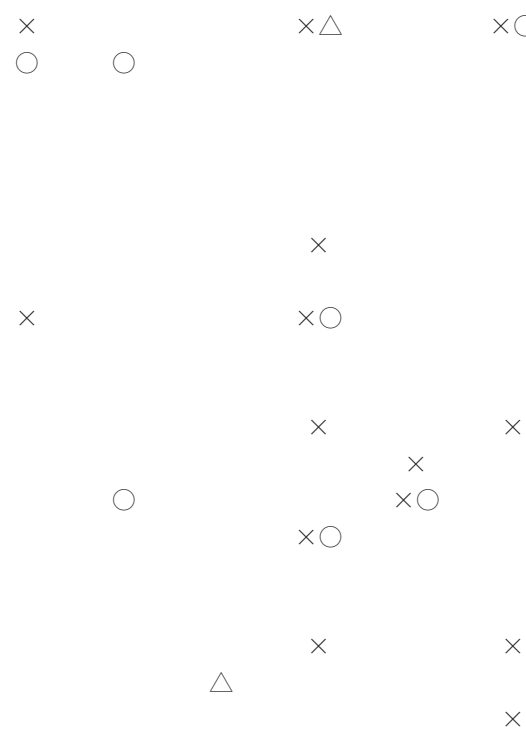

$\times$

(52) Miyako Is. (53) Irabu Is. (54) Shimoji Is. (55) Ishigaki Is. (56) Yonakuni Is. (57) Iriomote Is. (58) Kuro Is.(Yaeyama)

(59) Hateruma Is. (60) Hachijo Is. 
Table 1 Continued

$\bigcirc$, bottom sampling; $\triangle$, sweeping/sucking; $\times$, light sampling.

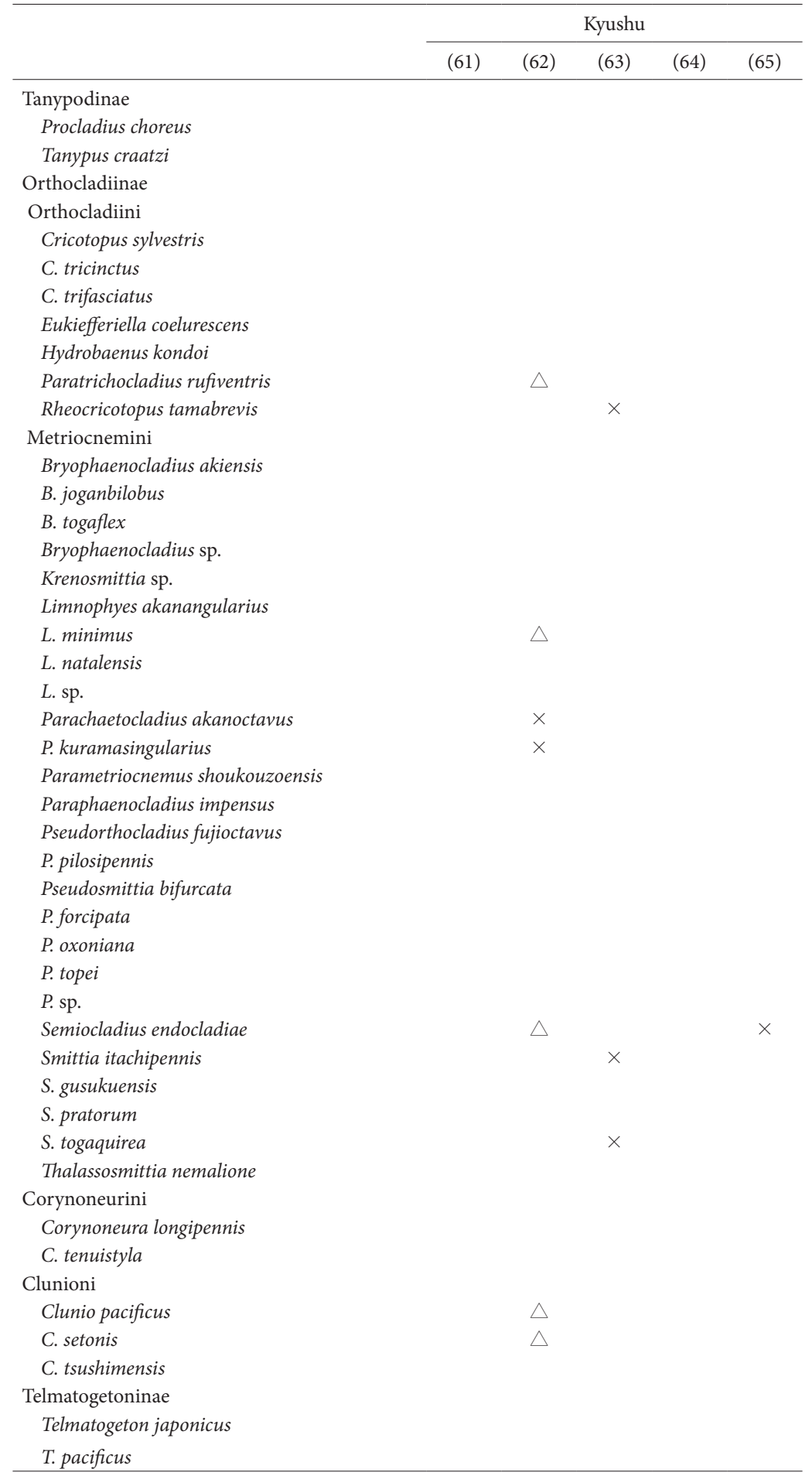

(61) Usuki (62) Nichinan (63) Sata (64) Shimabara (65) Ushibuka 
Table 1 Continued

$\bigcirc$, bottom sampling; $\triangle$, sweeping/sucking; $\times$, light sampling.

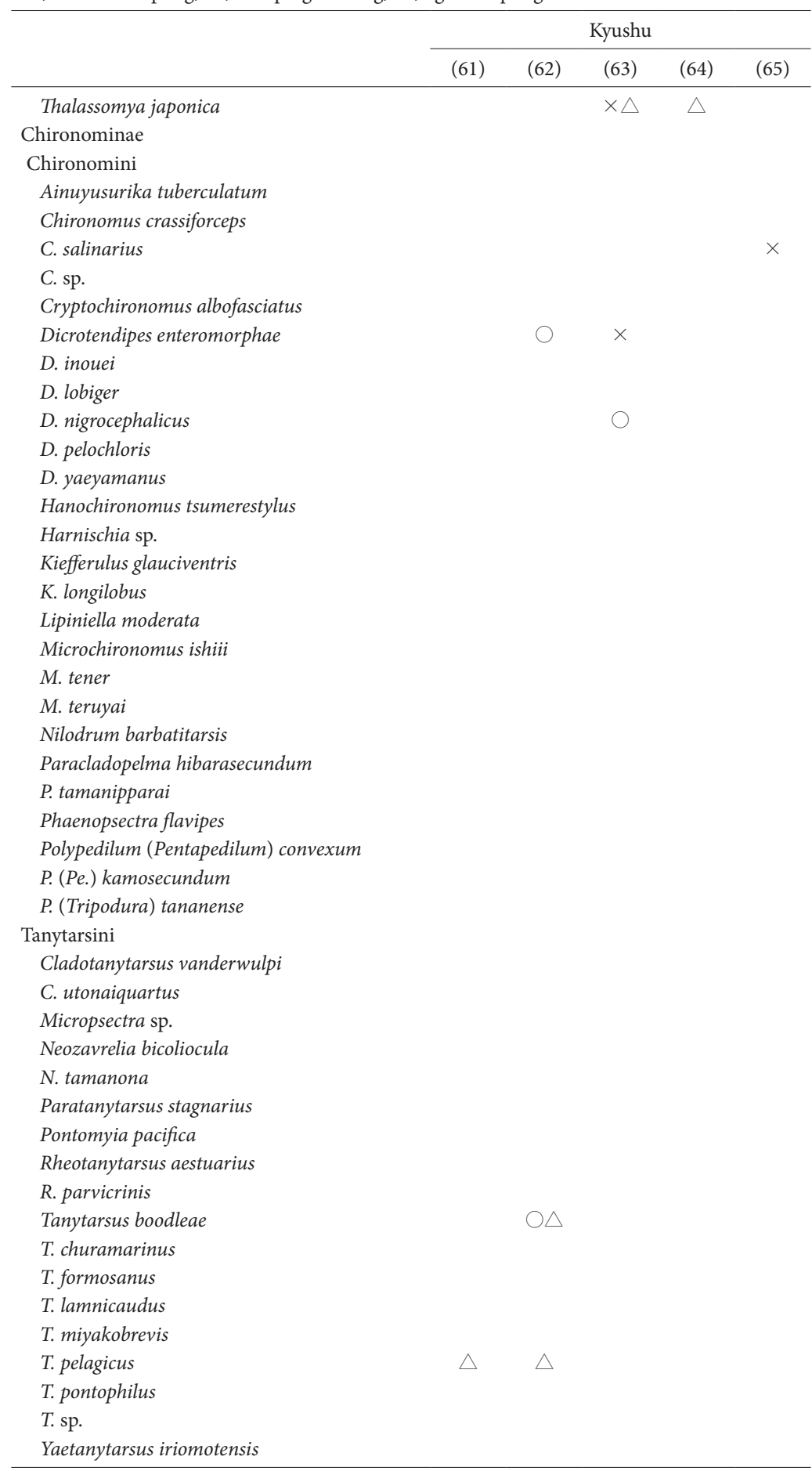

(61) Usuki (62) Nichinan (63) Sata (64) Shimabara (65) Ushibuka 
Table 1 Continued

\begin{tabular}{|c|c|c|}
\hline & \multicolumn{2}{|c|}{ References } \\
\hline & Freshwater & Brackish or marine \\
\hline \multicolumn{3}{|l|}{ Tanypodinae } \\
\hline Procladius choreus & Nihon Yusurika Kenkyu-kai (2010) & Sasa and Kikuchi (1995) \\
\hline Tanypus craatzi & Nihon Yusurika Kenkyu-kai (2010) & \\
\hline \multicolumn{3}{|l|}{ Orthocladiinae } \\
\hline \multicolumn{3}{|l|}{ Orthocladiini } \\
\hline Cricotopus sylvestris & Sasa and Kikuchi (1995) & Sasa and Kikuchi (1995) \\
\hline C. tricinctus & Sasa and Kikuchi (1995) & \\
\hline C. trifasciatus & Sasa and Kikuchi (1995) & \\
\hline Eukiefferiella coelurescens & Nihon Yusurika Kenkyu-kai (2010) & \\
\hline Hydrobaenus kondoi & Nihon Yusurika Kenkyu-kai (2010) & \\
\hline Paratrichocladius rufiventris & Nihon Yusurika Kenkyu-kai (2010) & \\
\hline Rheocricotopus tamabrevis & Nihon Yusurika Kenkyu-kai (2010) & \\
\hline \multicolumn{3}{|l|}{ Metriocnemini } \\
\hline Bryophaenocladius akiensis & Sasa and Kikuchi (1995) & \\
\hline B. joganbilobus & Sasa and Kikuchi (1995) & \\
\hline B. togaflex & Sasa and Kikuchi (1995) & \\
\hline \multicolumn{3}{|l|}{ Bryophaenocladius sp. } \\
\hline \multicolumn{3}{|l|}{ Krenosmittia sp. } \\
\hline Limnophyes akanangularius & Sasa and Kikuchi (1995) & \\
\hline L. minimus & Sasa and Kikuchi (1995) & Sasa and Kikuchi (1995) \\
\hline \multicolumn{3}{|l|}{ L. natalensis } \\
\hline \multicolumn{3}{|l|}{ L. sp. } \\
\hline Parachaetocladius akanoctavus & Sasa and Kikuchi (1995) & \\
\hline P. kuramasingularius & Sasa and Kikuchi (1995) & \\
\hline Parametriocnemus shoukouzoensis & Sasa and Kikuchi (1995) & \\
\hline Paraphaenocladius impensus & Sasa and Kikuchi (1995) & \\
\hline Pseudorthocladius fujioctavus & Sasa and Kikuchi (1995) & \\
\hline P. pilosipennis & Nihon Yusurika Kenkyu-kai (2010) & \\
\hline Pseudosmittia bifurcata & & Sasa and Kikuchi (1995) \\
\hline \multicolumn{3}{|l|}{ P. forcipata } \\
\hline \multicolumn{3}{|l|}{ P. oxoniana } \\
\hline \multicolumn{3}{|l|}{ P. topei } \\
\hline \multicolumn{3}{|l|}{ P. sp. } \\
\hline Semiocladius endocladiae & & Sasa and Kikuchi (1995) \\
\hline Smittia itachipennis & Sasa and Kikuchi (1995) & \\
\hline S. gusukuensis & & Sasa and Kikuchi (1995) \\
\hline S. pratorum & Sasa and Kikuchi (1995) & Sasa and Kikuchi (1995) \\
\hline S. togaquirea & Sasa and Kikuchi (1995) & \\
\hline Thalassosmittia nemalione & & Sasa and Kikuchi (1995) \\
\hline \multicolumn{3}{|l|}{ Corynoneurini } \\
\hline Corynoneura longipennis & Sasa and Kikuchi (1995) & \\
\hline C. tenuistyla & Sasa and Kikuchi (1995) & \\
\hline \multicolumn{3}{|l|}{ Clunioni } \\
\hline Clunio pacificus & & Sasa and Kikuchi (1995) \\
\hline C. setonis & & Sasa and Kikuchi (1995) \\
\hline C. tsushimensis & & Sasa and Kikuchi (1995) \\
\hline \multicolumn{3}{|l|}{ Telmatogetoninae } \\
\hline Telmatogeton japonicus & & Sasa and Kikuchi (1995) \\
\hline T. pacificus & & Sasa and Kikuchi (1995) \\
\hline
\end{tabular}


Table 1 Continued

\begin{tabular}{|c|c|c|}
\hline & \multicolumn{2}{|c|}{ References } \\
\hline & Freshwater & Brackish or marine \\
\hline Thalassomya japonica & & Sasa and Kikuchi (1995) \\
\hline \multicolumn{3}{|l|}{ Chironominae } \\
\hline \multicolumn{3}{|l|}{ Chironomini } \\
\hline Ainuyusurika tuberculatum & & Sasa and Kikuchi (1995) \\
\hline Chironomus crassiforceps & Sasa and Kikuchi (1995) & \\
\hline C. salinarius & & Sasa and Kikuchi (1995) \\
\hline \multicolumn{3}{|l|}{ C. sp. } \\
\hline Cryptochironomus albofasciatus & Nihon Yusurika Kenkyu-kai (2010) & \\
\hline Dicrotendipes enteromorphae & & Sasa and Kikuchi (1995) \\
\hline D. inouei & & Sasa and Kikuchi (1995) \\
\hline D. lobiger & Nihon Yusurika Kenkyu-kai (2010) & Sasa and Kikuchi (1995) \\
\hline D. nigrocephalicus & Nihon Yusurika Kenkyu-kai (2010) & \\
\hline D. pelochloris & Nihon Yusurika Kenkyu-kai (2010) & \\
\hline D. yaeyamanus & Sasa and Kikuchi (1995) & \\
\hline Hanochironomus tsumerestylus & Yamamoto et al., 2007 & Ree (1992) \\
\hline \multicolumn{3}{|l|}{ Harnischia sp. } \\
\hline Kiefferulus glauciventris & Nihon Yusurika Kenkyu-kai (2010) & \\
\hline K. longilobus & & Cranston, 2007 \\
\hline Lipiniella moderata & Nihon Yusurika Kenkyu-kai (2010) & \\
\hline Microchironomus ishiii & & Sasa and Kikuchi (1995) \\
\hline M. tener & Nihon Yusurika Kenkyu-kai (2010) & \\
\hline M. teruyai & Nihon Yusurika Kenkyu-kai (2010) & \\
\hline Nilodrum barbatitarsis & Nihon Yusurika Kenkyu-kai (2010) & \\
\hline Paracladopelma hibarasecundum & Sasa and Kikuchi (1995) & \\
\hline P. tamanipparai & Sasa and Kikuchi (1995) & \\
\hline Phaenopsectra flavipes & Sasa and Kikuchi (1995) & \\
\hline Polypedilum (Pentapedilum) convexum & Nihon Yusurika Kenkyu-kai (2010) & Cranston, 2007 \\
\hline P. (Pe.) kamosecundum & Sasa and Kikuchi (1995) & \\
\hline P. (Tripodura) tananense & Sasa and Kikuchi (1995) & Nihon Yusurika Kenkyu-kai (2010) \\
\hline \multicolumn{3}{|l|}{ Tanytarsini } \\
\hline Cladotanytarsus vanderwulpi & Sasa and Kikuchi (1995) & Sasa and Kikuchi (1995) \\
\hline C. utonaiquartus & Sasa and Kikuchi (1995) & \\
\hline \multicolumn{3}{|l|}{ Micropsectra sp. } \\
\hline Neozavrelia bicoliocula & Sasa and Kikuchi (1995) & \\
\hline N. tamanona & Sasa and Kikuchi (1995) & \\
\hline Paratanytarsus stagnarius & Nihon Yusurika Kenkyu-kai (2010) & \\
\hline Pontomyia pacifica & & Sasa and Kikuchi (1995) \\
\hline Rheotanytarsus aestuarius & Nihon Yusurika Kenkyu-kai (2010) & Nihon Yusurika Kenkyu-kai (2010) \\
\hline R. parvicrinis & Sasa and Kikuchi (1995) & \\
\hline Tanytarsus boodleae & & Sasa and Kikuchi (1995) \\
\hline T. churamarinus & & Sugimaru, Kawai et Imabayashi (2008) \\
\hline T. formosanus & & Sasa and Kikuchi (1995) \\
\hline T. lamnicaudus & & Tokunaga (1964) \\
\hline T. miyakobrevis & Sasa and Kikuchi (1995) & \\
\hline T. pelagicus & & Sasa and Kikuchi (1995) \\
\hline T. pontophilus & & Sasa and Kikuchi (1995) \\
\hline \multicolumn{3}{|l|}{ T. sp. } \\
\hline Yaetanytarsus iriomotensis & & Sasa and Kikuchi (1995) \\
\hline
\end{tabular}

